MATHEMATICS OF COMPUTATION

Volume 66, Number 220, October 1997, Pages 1561-1577

S 0025-5718(97)00858-2

\title{
A CONTINUITY PROPERTY OF MULTIVARIATE LAGRANGE INTERPOLATION
}

\author{
THOMAS BLOOM AND JEAN-PAUL CALVI
}

\begin{abstract}
Let $\left\{S_{t}\right\}$ be a sequence of interpolation schemes in $\mathbb{R}^{n}$ of degree $d$ (i.e. for each $S_{t}$ one has unique interpolation by a polynomial of total degree $\leq d)$ and total order $\leq l$. Suppose that the points of $S_{t}$ tend to $0 \in \mathbb{R}^{n}$ as $t \rightarrow$ $\infty$ and the Lagrange-Hermite interpolants, $H_{S_{t}}$, satisfy $\lim _{t \rightarrow \infty} H_{S_{t}}\left(x^{\alpha}\right)=0$ for all monomials $x^{\alpha}$ with $|\alpha|=d+1$. Theorem: $\lim _{t \rightarrow \infty} H_{S_{t}}(f)=T^{d}(f)$ for all functions $f$ of class $C^{l-1}$ in a neighborhood of 0 . (Here $T^{d}(f)$ denotes the Taylor series of $f$ at 0 to order $d$.)

Specific examples are given to show the optimality of this result.
\end{abstract}

\section{INTRODUCTION}

Let $O$ be an open neighborhood of the origin in $\mathbb{R}, a:=\left(a^{0}, \ldots, a^{d}\right) \in O^{d+1}$ and $f$ a function of class $C^{d+1}$ on $O$. As is well known, if $H\left[a^{0}, \ldots, a^{d}\right](f)$ denotes the Lagrange-Hermite interpolation polynomial with respect to the points $a^{0}, \ldots, a^{d}$ (with the usual convention when some points coincide), then

$$
\lim _{a \rightarrow 0} H\left[a^{0}, \ldots, a^{d}\right]=\mathcal{T}^{d} f
$$

where $\mathcal{T}^{d} f$ denotes the $d$-th Taylor polynomial of $f$ at the origin. This follows quite easily from the Newton representation formula for the interpolating polynomial, that is

$$
H\left[a^{0}, \ldots, a^{d}\right](f, x)=f\left(a^{0}\right)+\sum_{i=1}^{d} f\left[a^{0}, \ldots, a^{i}\right](f, x)\left(x-a^{0}\right) \ldots\left(x-a^{i-1}\right)
$$

via the Hermite-Genocchi formula for the divided differences, namely

$$
f\left[a^{0}, \ldots, a^{i}\right]=\int_{\Delta^{i}} f^{(i)}\left(a^{0}+\sum_{j=1}^{d} t_{j} a^{j}\right) d m(t)
$$

where $d m$ denotes Lebesgue measure on the simplex

$$
\Delta^{i}=\left\{\left(t_{j}\right)_{1 \leq j \leq i}: t_{j} \geq 0, \sum_{j=1}^{i} t_{j} \leq 1\right\} .
$$

More generally, for fixed $f$ of class $C^{d+k}$, one can prove similarly that the function $a \rightarrow H\left[a^{0}, \ldots, a^{d}\right](f)$ is of class $C^{k}$ on $O^{d+1}$ (see also [N, Th. 2.5]).

Received by the editor January 30, 1996 and, in revised form, August 21, 1996.

1991 Mathematics Subject Classification. Primary 41A05, 41A63.

Key words and phrases. Multivariable Lagrange interpolants, interpolation schemes in $\mathbb{R}^{n}$, Kergin interpolation.

The first author was supported by NSERC of Canada. 
The main purpose of this note is to study such continuity properties for multivariate Lagrange-Hermite interpolation.

For $x, y \in \mathbb{R}^{n}$ we write $|x|:=\sum_{i=1}^{n}\left|x_{i}\right|$ and $\langle x, y\rangle:=\sum_{i=1}^{n} x_{i} y_{i}$. The length of an $n$-multi-index $\alpha$ is denoted by $|\alpha|$, that is $|\alpha|=\sum_{i=1}^{n} \alpha_{i}$.

$\mathcal{P}_{n}^{d}=\mathcal{P}^{d}\left(\mathbb{R}^{n}\right)$ is the space of polynomials of $n$ real variables of degree at most $d$, $\mathcal{P}_{n}:=\bigcup_{d \geq 0} \mathcal{P}_{n}^{d}$ is endowed with the norm

$$
\|P\|_{\infty}:=\max _{|\alpha| \leq d}\left|c_{\alpha}\right|
$$

where $P=\sum_{|\alpha| \leq d} c_{\alpha} X^{\alpha}, X^{\alpha}$ being the polynomial function $x \rightarrow x^{\alpha}:=x_{1}^{\alpha_{1}} \ldots x_{n}^{\alpha_{n}}$. The functions $x \rightarrow x_{i}$ are simply denoted by $X_{i}$.

For a sufficiently differentiable function $f$, the partial derivatives are written as

$$
D^{\alpha} f=\partial^{\alpha} f / \partial x_{1}^{\alpha_{1}} \ldots \partial x_{n}^{\alpha_{n}} .
$$

We now define the multivariate Lagrange-Hermite interpolation polynomial (for general discussion, see $[\mathrm{L}])$.

Let $a^{1}, \ldots, a^{k}$ be $k$ points in $\mathbb{R}^{n}$ and let $S_{i}(i=1, \ldots, k)$ be $k$ directed sets of $n$-multi-indices of length not exceeding $d$ such that $\sum_{i=1}^{k} \# S_{i}=T(d, n)$. Here \# $S_{i}$ is the cardinality of $S_{i}, T(d, n):=\left(\begin{array}{c}n+d \\ d\end{array}\right)$ is the dimension of the space $\mathcal{P}_{n}^{d}$ and that $S_{i}$ is directed means that for every $\alpha \in S_{i}$ and every $\beta \leq \alpha$ (i.e., $\alpha_{j} \leq \beta_{j}$ for each $j$ ) we have $\beta \in S_{i}$.

We say that

$$
S:=\left\{\left(a^{1}, S_{1}\right), \ldots,\left(a^{k}, S_{k}\right)\right\}
$$

is an interpolation scheme of degree $d$ if, for every function $f$ defined, and with appropriate derivatives at the points $a^{i}$, there exists a unique polynomial $P \in \mathcal{P}_{n}^{d}$ such that

$$
D^{\alpha} P\left(a^{i}\right)=D^{\alpha} f\left(a^{i}\right), \quad \alpha \in S_{i} ; \quad i=1, \ldots, k .
$$

The polynomial $P$ is denoted by $H_{S}(f)$ and the points $a^{i}$ are sometimes referred to as nodes.

An interpolation scheme consisting of $T:=T(d, n)$ points (so that $\# S_{i}=1$ for each $i$ ) is called a unisolvent array of degree $d$, the corresponding interpolating polynomial is simply called the Lagrange interpolation polynomial of $f$ at $S$. It is usually denoted by $L_{S}(f)$. Note that $S=\left\{a^{1}, \ldots, a^{T}\right\}$ is unisolvent of degree $d$ if and only if it is not included in the zero set of a polynomial of degree not greater than $d$. Equivalently,

$$
\operatorname{det}\left(e_{\mu}\left(a^{i}\right)\right) \neq 0
$$

where $e_{\mu}(x)(1 \leq \mu \leq T)$ are the monomials of degree $\leq d$ ordered lexicographically. We shall use the notation $\operatorname{VDM}\left(a^{1}, a^{2}, \ldots, a^{T}\right)$ for $\operatorname{det}\left(e_{\mu}\left(a^{i}\right)\right)$ where VDM stands for Vandermonde. In this setting, the Lagrange formula is then

$$
L_{S}(f)=\sum_{i=1}^{T} f\left(a^{i}\right) l^{d i}(x),
$$

where $l^{d i}$ (or if necessary $l_{S}^{d i}$ ) is the Lagrange fundamental polynomial for $a^{i}$ defined by $l^{d i}\left(a^{j}\right)=1$ if $j=i$ and 0 otherwise $(j \leq T)$, that is

$$
l^{d i}(z)=\frac{V D M\left(a^{1}, \ldots, a^{i-1}, x, a^{i+1}, \ldots, a^{T}\right)}{V D M\left(a^{1}, \ldots, a^{i-1}, a^{i}, a^{i+1}, \ldots, a^{T}\right)} \quad(1 \leq i \leq T) .
$$


For specific examples of unisolvent arrays see [LP] and [Bo].

The Taylor polynomial to order $d$ at $a$, denoted $\mathcal{T}_{a}^{d} f$ is another example of a Lagrange-Hermite interpolation polynomial.

We can now state the general problem that is of interest in the multivariate context.

Problem 1.1. Let $S_{t}, t=1,2, \ldots$, be a sequence of interpolation schemes of degree $d$ whose points tend to 0 as $t \rightarrow \infty$ and $f$ a function sufficiently differentiable in a neighbourhood of 0 . Under what conditions is it true that $H_{S_{t}}(f)$ converges to $\mathcal{T}^{d} f\left(:=\mathcal{T}_{0}^{d} f\right)$ in $\mathcal{P}_{d}^{n}$ as $t$ approaches $\infty$ ?

Example 1.2 below shows that, in contrast to the one-variable case, smoothness conditions on $f$ do not, in general, guarantee a positive answer to 1.1.

Example 1.2. Let $S=\{(0,0),(u, v),(w, 0)\}$. It is a unisolvent array of degree 1 in $\mathbb{R}^{2}$ whenever $v w \neq 0$. We have $L_{S}\left(x_{1}^{2}, x\right)=w x_{1}-\frac{u(w-u)}{v} x_{2}$. If we take $a, b, c>0$ with $c>a, b>2 a$ and $u=u_{t}=t^{-a}, v=v_{t}=t^{-b}, w=w_{t}=t^{-c}$, one sees that the coefficient of $x_{2}$ in $L_{S_{t}}\left(x_{1}^{2}, x\right)$ tends to $\infty$ as $t \rightarrow \infty$.

The paper is organized as follows.

In section 2 we briefly collect some earlier results related to 1.1.

Section 3 contains our main theorem which provides a criterion of different nature.

Most of the calculations are postponed to section 4 where a quantitative version of the main theorem, using somewhat more technical tools, is presented.

The results are illustrated by examples in the final section.

\section{EARLier RESUlts AND BASIC CRITERIA}

The following proposition is an immediate consequence of Taylor's Theorem. Its proof will be omitted. To simplify matters, we state it only for Lagrange interpolation and unisolvent arrays.

Proposition 2.1. Let $S_{t}=\left\{a^{1 t}, \ldots, a^{T t}\right\}$ be a sequence of unisolvent arrays of degree $d$ in $\mathbb{R}^{n}$. We write $l_{t}^{d i}$ for $l_{S_{t}}^{d i}$ and $L_{t}$ for $L_{S_{t}}$. If for $1 \leq i \leq T$ we have

$$
\lim _{t \rightarrow \infty}\left|a^{i t}\right|^{d+1}|| l_{t}^{d i} \|_{\infty}=0
$$

then for every function $f$ of class $C^{d+1}$ in a neighborhood of 0 ,

$$
L_{t} f \rightarrow \mathcal{T}^{d} f, \quad t \rightarrow \infty .
$$

Even in the one-variable case, (2.1) is sufficient but not necessary for the conclusion of Proposition 2.1 to hold. It can however be applied to some interesting sequences of arrays. They are constructed as follows. Let $S=\left\{a^{1}, \ldots, a^{T}\right\}$ be a unisolvent array of degree $d$ in $\mathbb{R}^{n}$ and let $A_{t}$ be a sequence of linear automorphisms of $\mathbb{R}^{n}$. Then $S_{t}=A_{t}(S):=\left\{A_{t}\left(a^{1}\right), \ldots, A_{t}\left(a^{T}\right)\right\}$ is again unisolvent.

Corollary 2.2. Assume that $\left\|A_{t}\right\|^{d+1}\left\|A_{t}^{-1}\right\|^{d} \rightarrow 0$ as $t \rightarrow \infty$, where $\|\cdot\|$ is any matrix norm. Then $L_{S_{t}} f \rightarrow \mathcal{T}^{d} f$ for every $f$ of class $C^{d+1}$ in a neighborhood of 0 .

Proof. First we verify easily that $l_{t}^{d i}=l_{S}^{d i} \circ A_{t}^{-1}$, therefore if $l_{S}^{d i}(x)=\sum_{|\alpha| \leq d} c_{\alpha} X^{\alpha}$, it follows that

$$
\left\|l_{t}^{d i}\right\|_{\infty} \leq\left\|l_{S}^{d i}\right\|_{\infty} \sum_{|\alpha| \leq d}\left\|X^{\alpha} \circ A_{t}^{-1}\right\|_{\infty}
$$


But a computation shows that

$$
\left\|X^{\alpha} \circ A_{t}^{-1}\right\|_{\infty}=O\left(\left\|A_{t}^{-1}\right\|^{|\alpha|}\right)=O\left(\left\|A_{t}^{-1}\right\|^{d}\right) .
$$

The last equality holds since, by the hypothesis, we necessarily have $\left\|A_{t}\right\|^{-1} \geq 1$ for $t$ large. We deduce that

$$
\left|a^{i t}\right|^{d+1} \cdot\left\|l_{t}^{d i}\right\|_{\infty}=O\left(\left\|A_{t}\right\|^{d+1} \cdot\left\|A_{t}^{-1}\right\|^{d}\right) \rightarrow 0,
$$

which shows that the condition (2.1) of Proposition 2.1 is satisfied.

Coatmelec, [Co, Th II.2.5], first investigated conditions on such sequences of unisolvent arrays. He gave a different but rather technical condition-which is however proved to be satisfied when the $A_{t}$ are scalings by ratio $\rho_{t}\left(\lim _{t \rightarrow \infty} \rho_{t}=0\right)$ composed with a rotation $R_{t}$ - that ensures convergence to the Taylor polynomial for functions of class $C^{d}$.

Corollary 2.2. is implied by the results of a paper by Ciarlet and Raviart, [CR], where one also finds a nice geometric property that implies (2.1). Let us just state it in case $d=1,(2.1)$ holds if the quotient of the circumradius and the innerradius of the simplex with vertices $A_{t}\left(a^{i}\right), i=1, \ldots, n+1$, remains bounded as $t \rightarrow \infty$ which means, in other words, that the simplex of vertices the nodes of $S_{t}$ does not become more and more flat.

The following example shows that there exist sequences $\left(A_{t}\right)$ that work for every function of class $C^{d+1}$ but not, in general, for functions only of class $C^{d}$.

Example 2.3. We take

$$
S_{t}=\left\{(0,0),\left(1 / t^{2}, 1 / t^{3}\right),\left(2 / t^{2}, 0\right)\right\}=A_{t}(S)
$$

with $S=\{(0,0),(1,0),(0,1)\}$. Using the matrix norm given by the maximum of the absolute value of the coefficients, we get

$$
A_{t}=\left(\begin{array}{cc}
1 / t^{2} & 2 / t^{2} \\
1 / t^{3} & 0
\end{array}\right) \Longrightarrow\left\|A_{t}\right\|=2 / t^{2}, \quad\left\|A_{t}^{-1}\right\|=t^{3}
$$

so that

$$
\left\|A_{t}\right\|^{2}\left\|A_{t}^{-1}\right\|=4 / t
$$

and by $2.2, L_{S_{t}}(f) \rightarrow \mathcal{T}^{1}(f)$ for every function $f$ of class $C^{2}$. However, the function $f$ defined by $f(x)=x^{3 / 2}$ if $x \geq 0$ and 0 otherwise, is of class $C^{1}$ on $\mathbb{R}^{2}$ while

$$
L_{S_{t}}(f, x)=\frac{\sqrt{2}}{t} x_{1}-(\sqrt{2}-1) x_{2}
$$

in which the $x_{2}$-coefficient does not tend to 0 . Thus $L_{S_{t}}(f)$ does not converge to the Taylor polynomial of $f$.

Thus in contrast to the one-variable case, the degree of differentiability needed to guarantee a positive answer to 1.1 may depend on the sequence of schemes.

We shall give in the last section an example (Example 5.4) not satisfying (2.1) but for which convergence to the Taylor polynomial holds in general, for functions whose degree of differentiability is greater than $d+1$. We provide also in our main theorem in section 3 an upper bound for the level of differentiability that may be necessary in order to get the convergence.

Finally, let us mention an important class of arrays that reproduce the same continuity properties as for dimension 1 . We briefly describe these arrays in $\mathbb{R}^{2}$ (for notational simplicity) and explain the phenomenon. 
Example 2.4. We work in $\mathbb{R}^{2}$. Let $d \geq 1$ and for $s=1,2,3, \ldots$ let ${ }_{t} A$ and ${ }_{t} B$ be two sequences of $d+1$ pairwise distinct real numbers. Their index set is assumed to begin at 0 . Next we define

$$
S_{t}:=\left\{\left({ }_{t} A_{i},{ }_{t} B_{j}\right), \quad i+j \leq d\right\} .
$$

Then $S_{t}$ is a unisolvent array of order $d$. Moreover if ${ }_{t} A$ and ${ }_{t} B$ tend to 0 as $t \rightarrow \infty$, then $L_{s_{t}} f$ converges to $\mathcal{T}^{d} f$ for every $f$ of class $C^{d}$ in a neighborhood of the origin.

A Newton formula is available for the interpolants corresponding to this array (note that it is strongly dependent on the ordering of the sequences $A$ and $B$ ). If $f$ is defined in a neighbourhood of 0 , we have (dropping the " $t$ ")

$$
L_{S}(f, x)=\sum_{i+j \leq d} a_{i j}\left(x_{1}-A_{0}\right) \ldots\left(x_{1}-A_{i-1}\right)\left(x_{2}-B_{0}\right) \ldots\left(x_{2}-B_{j-1}\right)
$$

where the $a_{i j}$ 's are bivariate divided differences defined as follows. The one dimensional divided difference $f\left(., x_{2}\right)\left[A_{0}, \ldots, A_{i}\right]$ is a function of $x_{2}$, say $g_{i}$, then $a_{i j}=g_{i}\left[B_{0}, \ldots, B_{j}\right]$. The two variables actually play a symmetric role.

Therefore the claim on the convergence follows essentially as in the one-variable case.

\section{MAIN THEOREM}

We shall first define some other quantities attached to an interpolation scheme that will naturally come into play in our main Theorem 3.3. Let

$$
S=\left\{\left(a^{1}, S_{1}\right), \ldots,\left(a^{k}, S_{k}\right)\right\}
$$

be an interpolation scheme of degree $d$ in $\mathbb{R}^{n}$, we shall write $a^{i} \in S$ and $|S|:=$ $\max \left\{\left|a^{i}\right|, i=1, \ldots, k\right\}$.

Definition 3.1. The order - denoted by $o\left(a^{i}\right)$ — of $a^{i}$ for $S$ is defined by $o\left(a^{i}\right):=$ $\max \left\{|\alpha|+1, \quad \alpha \in S_{i}\right\}$ and the total order of $S$ is $o(S):=\sum_{i=1}^{k} o\left(a^{i}\right)$.

Thus for example, if $S$ is a unisolvent array of degree $d$, then its total order is $T(d, n)$ while if $S$ is only a Taylor scheme, its total order is $d+1$. Actually we have the following simple

Lemma 3.2. For every interpolation scheme $S$ of degree $d$ in $\mathbb{R}^{n}$ we have $d+1 \leq$ $o(S) \leq T(d, n)$.

Proof. We use the notation (3.1) for $S$. To get the upper bound, it is obviously enough to prove that $o\left(a^{i}\right) \leq \# S_{i}$ for every $i$. This follows readily from the simple inequality

$$
\alpha_{1}+\alpha_{2}+\cdots+\alpha_{n}+1 \leq\left(\alpha_{1}+1\right)\left(\alpha_{2}+1\right) \ldots\left(\alpha_{n}+1\right),
$$

together with the observation that if $\alpha \in S_{i}$ with $|\alpha|=o\left(a^{i}\right)-1$, then since $S_{i}$ is directed, contains at least $\left(\alpha_{1}+1\right)\left(\alpha_{2}+1\right) \ldots\left(\alpha_{n}+1\right)$ elements.

Next, let us write $d_{i}=o\left(a^{i}\right)-1$. We prove that $d_{1}+\cdots+d_{k} \geq d$ from which the lower bound immediately follows. We have

$$
\begin{aligned}
\left(\begin{array}{c}
d+n \\
n
\end{array}\right) & =\sum_{i=1}^{k} \# S_{i} \leq \sum_{i=1}^{k}\left(\begin{array}{c}
d_{i}+n \\
n
\end{array}\right)=\sum_{i=1}^{k} \sum_{j=0}^{n}\left(\begin{array}{c}
n \\
j
\end{array}\right)\left(\begin{array}{c}
d_{i} \\
n-j
\end{array}\right) \\
& \leq \sum_{j=0}^{n}\left(\begin{array}{c}
n \\
j
\end{array}\right)\left(\begin{array}{c}
d_{1}+\cdots+d_{k} \\
n-j
\end{array}\right)=\left(\begin{array}{c}
d_{1}+\cdots+d_{k}+n \\
n
\end{array}\right)
\end{aligned}
$$

which implies $d \leq d_{1}+\cdots+d_{k}$. 
Note that the proof shows also that $o(S)=d+1$ if and only if $S$ is a Taylor scheme and $o(S)=T(d, n)$ if and only if $S$ is a unisolvent array of degree $d$.

Theorem 3.3. Let $S_{t}$ be a sequence of interpolation schemes of degree $d$ in $\mathbb{R}^{n}$. $W e$ assume that for $t$ large $o\left(S_{t}\right) \leq l$. If the following condition holds

$$
|\alpha|=d+1 \Longrightarrow \lim _{t \rightarrow \infty} H_{S_{t}} X^{\alpha}=0,
$$

then for every function $f$ of class $C^{l-1}$ in a neighborhood of the origin we have

$$
\lim _{t \rightarrow \infty} H_{S_{t}} f=\mathcal{T}^{d} f .
$$

The next lemma shows that the points in such sequences of schemes must tend to zero as $t \rightarrow \infty$ so that the theorem deals indeed with Problem 1.1. It also shows that the statement of Theorem 3.3 is meaningful as soon as $f$ is defined in any neighborhood of the origin.

Lemma 3.4. Under the hypothesis of Theorem 3.3, we have $\lim _{t \rightarrow \infty}\left|S_{t}\right|=0$.

When there is no danger of confusion, we write $H_{t} f$ for $H_{S_{t}} f$ and define the coefficients ${ }_{t} C_{\beta}\left(X^{\alpha}\right)$ by

$$
H_{t}\left(X^{\alpha}\right)=\sum_{|\beta| \leq d}{ }_{t} C_{\beta}\left(X^{\alpha}\right) X^{\beta} .
$$

Thus, hypothesis (3.2) means that for every $\beta$ and every $\alpha$ such that $|\beta| \leq d$ and $|\alpha|=d+1$, we have

$$
\lim _{t \rightarrow \infty} C_{\beta}\left(X^{\alpha}\right)=0
$$

Proof of 3.4. Supposing that $\left|S_{t}\right|$ is unbounded, there exists a subsequence of points $a^{t_{k}} \in S_{t_{k}}$ such that

$$
\lim _{k \rightarrow \infty}\left|a^{t_{k}}\right|=+\infty
$$

In particular we may assume that $\left|a^{t_{k}}\right|>n$. For every $k$ we take an index $j \in$ $\{1, \ldots, n\}$ such that $\left|a_{j}^{t_{k}}\right|$ is maximal and therefore greater than 1 . Then we have,

$$
\begin{aligned}
& \left(a_{j}^{t_{k}}\right)^{d+1}=H_{t_{k}}\left(X_{j}^{d+1}\right)\left(a^{t_{k}}\right)=\sum_{|\beta| \leq d} t_{k} C_{\beta}\left(X_{j}^{d+1}\right) X^{\beta}\left(a^{t_{k}}\right) \\
\Longrightarrow & \left|a_{j}^{t_{k}}\right|^{d+1} \leq\left[\sum_{|\beta| \leq d} \mid t_{k} C_{\beta}\left(X_{j}^{d+1}\right)\right] \times \times\left|a_{j}^{t_{k}}\right|^{d} \\
\Longrightarrow & \left|a_{j}^{t_{k}}\right| \leq \sum_{|\beta| \leq d}\left|t_{k} C_{\beta}\left(X_{j}^{d+1}\right)\right| \rightarrow 0 \quad(k \rightarrow \infty)
\end{aligned}
$$

which is a contradiction. Consequently the sequence $\left|S_{t}\right|$ is bounded. Now, using the same estimates again, we can show that $\left|S_{t}\right|$ converges to 0 .

Though it states a very simple and elementary algebraic property satisfied by Lagrange-Hermite interpolants, the next Lemma 3.5 is an essential element of Theorem 3.3. This property will be used in Lemma 3.6 to prove that, under the assumption (3.2), the convergence (3.3) holds for every polynomial. 
Lemma 3.5. Let $S$ be an interpolation scheme of degree $d$ in $\mathbb{R}^{n}$. Then for every polynomial $P$ and $Q$ we have

$$
H_{S}(P Q)=H_{S}\left(P H_{S}(Q)\right) .
$$

Proof. The verification is straightforward. We assume that $S$ is as in (3.1). Then, due to the uniqueness of the interpolation polynomial, it suffices to show that for every $i \in\{1, \ldots, k\}$ and every $\alpha \in S_{i}$ we have

$$
D^{\alpha}(P Q)\left(a^{i}\right)=D^{\alpha}\left(P H_{S} Q\right)\left(a^{i}\right) .
$$

This follows from the multivariate Leibniz formula, using the fact that $S_{i}$ is directed, for

$$
\begin{aligned}
D^{\alpha}\left(P H_{S} Q\right)\left(a^{i}\right) & =\sum_{\beta \leq \alpha}\left(\begin{array}{c}
\alpha \\
\beta
\end{array}\right) D^{\beta}\left(H_{S} Q\right)\left(a^{i}\right) D^{\alpha-\beta} P\left(a^{i}\right) \\
& =\sum_{\beta \leq \alpha}\left(\begin{array}{c}
\alpha \\
\beta
\end{array}\right) D^{\beta} Q\left(a^{i}\right) D^{\alpha-\beta} P\left(a^{i}\right) \\
& =D^{\alpha}(P Q)\left(a^{i}\right) .
\end{aligned}
$$

Lemma 3.6. Under the hypothesis of Theorem 3.3, for every polynomial P, we have

$$
\lim _{t \rightarrow \infty} H_{t} P=\mathcal{T}^{d} P
$$

Thus for every $m \in \mathbb{N}, H_{t}$ converges to $\mathcal{T}^{d}$ as a sequence of (continuous) operators on $\mathcal{P}_{n}^{m}$.

Proof. Since $H_{t} P=P$ when the degree of $P$ is not greater than $d$, it suffices to show that for every $k \geq 1$, every $\alpha$ such that $|\alpha|=d+k$ we have $H_{t}\left(X^{\alpha}\right) \rightarrow \mathcal{T}^{d}\left(X^{\alpha}\right)=0$. We will prove by induction on $|\alpha|$. For $k=1$, it is the hypothesis (3.2). We assume that the property is true for $k \leq l$ and will prove it for $l+1$. Thus with the notation (3.4), we have

$$
(|\delta| \leq d, \quad d<|\alpha| \leq d+l) \Longrightarrow{ }_{t} C_{\delta}\left(X^{\alpha}\right) \rightarrow 0 \quad(t \rightarrow \infty) .
$$

Now, take $\alpha^{\prime}$ such that $\left|\alpha^{\prime}\right|=d+l+1$. Without loss of generality we may suppose that $X^{\alpha^{\prime}}=X_{1} X^{\alpha}$ with $|\alpha|=d+l$. Therefore, using Lemma 3.5, we have

$$
\begin{aligned}
H_{t} X^{\alpha^{\prime}} & =H_{t}\left(X_{1} X^{\alpha}\right) \\
& =H_{t}\left(X_{1} H_{t} X^{\alpha}\right) \\
& =H_{t}\left(X_{1} \sum_{|\delta| \leq d}{ }_{t} C_{\delta}\left(X^{\alpha}\right) X^{\delta}\right) \\
& =\sum_{|\delta| \leq d}{ }_{t} C_{\delta}\left(X^{\alpha}\right) H_{t}\left(X_{1} X^{\delta}\right) \\
& =\sum_{|\delta|<d}{ }_{t} C_{\delta}\left(X^{\alpha}\right) X_{1} X^{\delta}+\sum_{|\delta|=d}\left\{{ }_{t} C_{\delta}\left(X^{\alpha}\right) \sum_{|\tilde{\delta}| \leq d}{ }_{t} C_{\tilde{\delta}}\left(X_{1} X^{\delta}\right) X^{\tilde{\delta}}\right\} .
\end{aligned}
$$

Now, using (3.5), all the coefficients in (3.6) tend to zero as $t \rightarrow \infty$.

The idea of the proof of Theorem 3.3 is quite simple. Roughly, we will replace the function $f$ by a polynomial $P=P_{t}$ which satisfies the interpolation conditions and has appropriate behaviour as a function of the nodes. In this case we will have 
$H_{t}(f)=H_{t}(P)$ and the latter can be handled with the previous Lemma. Here the polynomial $P$ will be a Kergin interpolation polynomial (see also Theorem $6.1[\mathrm{~K}]$ ).

We now recall briefly the main properties of Kergin interpolation that we shall need (see e.g $[\mathrm{M}]$ ).

Let $\Omega$ be a convex subset of $\mathbb{R}^{n}$ and $Y:=\left\{y^{0}, \ldots, y^{d}\right\}$, a subset of $d+1$ not necessarily distinct points in $\Omega$. Then the Kergin interpolation polynomial $K_{Y}(f)=$ $K\left[y^{0}, \ldots, y^{d}\right](f)$ (or $K f$ for short) is defined for every function $f$ of class $C^{d}$ on $\Omega$ by the formula (using the directional derivative notation $D_{u} f(a):=D f(a) . u$ )

$$
K f(x)=\sum_{i=0}^{d} \int_{\Delta^{i}} D_{x-y^{0}} D_{x-y^{1}} \ldots D_{x-y^{i-1}} f\left(y^{0}+\sum_{i=1}^{d} t_{j}\left(y^{j}-y^{0}\right)\right) d m(t) .
$$

(i) It is a polynomial of degree at most $d$ that interpolates $f$ at the point $y^{i}$. If some point is repeated, say $y_{1}$ three times, we have $D^{j} f\left(y^{1}\right)=D^{j}(K f)\left(y^{1}\right)$ for $j=0,1,2$ where $D^{j} f\left(y^{1}\right)$ denotes the (total) $j$-th derivatives of $f$ at $y^{1}$. In particular when all the points coincide then $K f$ is the Taylor polynomial of $f$ to the order $d$.

(ii) If $f$ is of the form $f(x)=g(\langle x, z\rangle)$ with $g$ a function of one variable, then

$$
K\left[y^{0}, \ldots, y^{d}\right](f, x)=H\left[\left\langle y^{0}, z\right\rangle, \ldots,\left\langle y^{d}, z\right\rangle\right](g,\langle x, z\rangle) .
$$

This is the fundamental property of Kergin interpolation.

(iii) It follows from $(3.7)$ that $K\left[y^{0}, y^{1}, \ldots, y^{d}\right](f)$ is a continuous function of the points $y^{i}$.

(iv) $K f$ is independent of the order of the points.

(v) If $\left\{w^{i}, i=0, \ldots, k\right\} \subset\left\{y^{j}, j=0, \ldots, d\right\}$, then

$$
\left(K\left[w^{0}, \ldots, w^{k}\right] \circ\left(K_{Y}(f)\right)=K\left[w^{0}, \ldots, w^{k}\right](f)\right.
$$

for every $f$ of class $C^{d}$ on $\Omega$.

Proof of 3.3. Let $f$ be a function of class $C^{l-1}$ in some neighborhood $\Omega$ of the origin that we may suppose to be convex. We shall define a subset $Y_{t}$ of $o\left(S_{t}\right)$ points in $\Omega$ as follows: $a \in Y_{t}$ if $a \in S_{t}$ and furthermore it is repeated $o(a)$ times. Thus we have indeed $\# Y_{t}=\sum_{a \in S_{t}} o(a)=o\left(S_{t}\right)$.

Let us consider the Kergin interpolation polynomial $K_{Y_{t}} f$ (or $K_{t} f$ ). Its degree is at most $o\left(S_{t}\right)-1$. For $t$ large enough, this polynomial is well defined since $o\left(S_{t}\right) \leq l$ and $f$ is $l-1$ times continuously differentiable. Actually, this will be the only point where the full smoothness of $f$ will be used. Now the polynomial $K_{t}$ satisfies

$$
D^{j} f(a)=D^{j} K_{t}(f)(a) \quad\left(a \in S_{t} ; 1 \leq j \leq o(a)-1\right)
$$

so that

$$
H_{t} f=H_{t}\left(K_{t} f\right)
$$

Therefore we have

$$
H_{t} f-\mathcal{T}^{d} f=\left[H_{t}\left(K_{t} f\right)-\mathcal{T}^{d}\left(K_{t} f\right)\right]+\left[\mathcal{T}^{d}\left(K_{t} f\right)-\mathcal{T}^{d} f\right] .
$$

We shall prove that

1. $\lim _{t \rightarrow \infty} \mathcal{T}^{d}\left(K_{t} f\right)=\mathcal{T}^{d} f$

2. $\lim _{t \rightarrow \infty} H_{t}\left(K_{t} f\right)-\mathcal{T}^{d}\left(K_{t} f\right)=0$. 
Let $W_{t}$ be a subset of $Y_{t}$ consisting of $(d+1)$ points (by Lemma $3.2 \# Y_{t} \geq d+1$ ). Then, by Lemma 3.4, all the points in $W_{t}$ tend to 0 as $t \rightarrow \infty$. Therefore, due to the known continuity properties of the Kergin interpolant, $\tilde{K}_{t}:=K_{W_{t}}$ converges to $\mathcal{T}^{d}$ as a sequence of operators on $\mathcal{P}_{n}^{m}$ for each $m \geq d$. On the other hand, again by Lemma 3.4 the sequence $K_{t} f$ is bounded in $\mathcal{P}_{n}^{l-1}$ ( see the formula (3.7) for Kergin interpolants). Consequently $\lim _{t \rightarrow \infty} \mathcal{T}^{d}\left(K_{t} f\right)-\tilde{K}_{t}\left(K_{t} f\right)=0$. Now, using that $W_{t} \subset Y_{t}$ and property (3.8) we get

$$
\begin{aligned}
\mathcal{T}^{d}\left(K_{t} f\right)-\mathcal{T}^{d} f & =\mathcal{T}^{d}\left(K_{t} f\right)-\tilde{K}_{t}\left(K_{t} f\right)+\tilde{K}_{t}\left(K_{t} f\right)-\mathcal{T}^{d} f \\
& =\left[T^{d}\left(K_{t} f\right)-\tilde{K}_{t}\left(K_{t} f\right)\right]+\left[\tilde{K}_{t} f-\mathcal{T}^{d} f\right] .
\end{aligned}
$$

But both terms in parentheses above tend to 0 so that the limit (1) holds.

The limit (2) follows similarly from the boundedness of the sequence $K_{t} f$ in $\mathcal{P}_{n}^{l-1}$ since, by Lemma 3.6, $H_{t}$ converges to $\mathcal{T}^{d}$ as a sequence of operators on $\mathcal{P}_{n}^{l-1}$. The theorem is proved.

The conclusion of Theorem 3.3 is not valid, in general, if $f$ is only of class $\mathcal{C}^{d+1}$. Example 5.1 is a specific sequence of schemes where the degree of differentiability required in Theorem 3.3 is optimal.

We note that, in the particular case of a sequence of unisolvent arrays, the Kergin interpolants that are used, in an essential way, in the proof could be replaced by the interpolation polynomials exhibited in Example 2.4. Precisely, working in $\mathbb{R}^{2}$, if $S_{t}:=\left\{a^{1 t}, \ldots, a^{T t}\right\}$ we can use in place of $K_{t} f$ the interpolation polynomials (of degree $T-1)$ constructed from the sequences

$$
{ }_{t} A:=\left\{a_{1}^{1 t}, \ldots, a_{2}^{T t}\right\} \quad \text { and } \quad{ }_{t} B:=\left\{a_{2}^{T t}, a_{2}^{T-1 t}, \ldots, a_{2}^{1 t}\right\} .
$$

However these polynomials are not well adapted for use in the case of general Lagrange-Hermite schemes.

In the next section, we shall see that, with more computations, it will be possible to use the proof of Theorem 3.3, to obtain specific estimates on the way that the quantity

$$
M_{S}:=\sup \left\{\left\|H_{S}\left(X^{\alpha}\right)\right\|_{\infty},|\alpha|=d+1\right\}
$$

bounds the approximation error $\left\|H_{S} f-\mathcal{T}^{d} f\right\|_{\infty}$.

An easy generalization of Theorem 3.3 (but less precise) is the following: Let $S_{t}$ be a sequence of schemes of degree $d$ and of total order not greater than $l$ and let $T$ be another scheme of same degree. Then if $H_{S_{t}} P$ converges to $H_{T} P$ for every monomial $P$ of degree $d+1$, we have as well

$$
\lim _{t \rightarrow \infty} H_{S_{t}} f=H_{T} f
$$

for every function $f$ of class $C^{l+o(T)-1}$ on a convex open set containing all the nodes in the $S_{t}$ and $T$. The proof can be carried out as for 3.3 but in considering the Kergin interpolation polynomials $K_{Y_{t}}$ with $a \in Y_{t}$ if $a \in S_{t}$ or $a \in T$, and in each case $a$ is repeated $o(a)$ times (if $a$ belongs to both schemes, we take the largest order). The end of the proof is even simpler for the second term in the right-hand side of (3.10), in which $\mathcal{T}^{d} f$ is replaced by $H_{T} f$, now vanishes.

Finally, we note that the number of "test" functions in hypothesis (3.2) of Theorem 3.3 cannot be reduced, in general, as Example 1.2 shows. 


\section{Quantitative estimates}

For an $m$ times continuously differentiable function $f$ on a neighborhood of a compact set $K$, we define the semi-norm

$$
\|f\|_{m, K}:=\sup _{0 \leq j \leq m}\left\{\sup _{x \in K}\|\| D^{j} f(x)\|\|\right\}
$$

where \|\|$. \| \mid$ is the standard norm for $j$-multi-linear forms (when $\mathbb{R}^{n}$ is endowed with the norm $|x|$ ). The purpose of this section is to study the following

Problem 4.1. Let $S$ be an interpolation scheme of degree $d$ in $\mathbb{R}^{n}, C v(S)$ denotes the convex hull of the set formed of nodes in $S$ and the origin, and let $f$ be a function of class $C^{l}$ in a neighborhood of $C v(S)$. Find a bound for $\left\|H_{S} f-\mathcal{T}^{d} f\right\|_{\infty}$ that makes use only of $M_{S}(\operatorname{see}(3.12)),|S|$, and $\|f\|_{o(S)}=\|f\|_{o(S), C v(S)}$.

We will assume that

$$
M_{S} \leq 1 \quad \text { and } \quad|S| \leq 1
$$

We shall state the estimates with the Landau " $O$ " notation. If the constants involved depend only on, say, $d$ and $n$, we will write $O_{d, n}$.

We have

Theorem 4.1. Let $S$ be an interpolation scheme of degree $d$ in $\mathbb{R}^{n}$, of total order $l$ and satisfying $(\star)$. Then for every function $f$ of class $C^{l}$ in a neighbourhood of $C v(S)$ we have

$$
\left\|H_{S} f-\mathcal{T}^{d} f\right\|_{\infty}=O\left(|S|+M_{S}\right) .\|f\|_{l}
$$

To prove it, we shall just examine in detail each term appearing in the proof of Theorem 3.3. Thus Lemma 4.2 is a quantitative version of Lemma 3.6 and Lemma 4.3 establishes some further properties of Kergin interpolation. It seems to be of interest in itself (see also [W] and [SX] ).

Lemma 4.2. Let $S$ be an interpolation scheme of degree $d$ satisfying $(\star)$ and $m \geq$ $d+1$. Then for every polynomial $P \in \mathcal{P}_{n}^{m}$ we have

$$
\left\|H_{S} P-\mathcal{T}^{d} P\right\|_{\infty} \leq O_{d, m, n}\left(M_{S}\right) \cdot\|P\|_{\infty}
$$

Proof. The result is an easy consequence of the following. Let $k \geq 0$, we have $\max _{|\beta|=d+k+1}\left\|H_{S}\left(X^{\beta}\right)\right\|_{\infty} \leq M_{k}$ where $M_{k}$ is the sequence defined recursively by $M_{0}:=M_{S}$ and $M_{k+1}:=\mu M_{k}$ with $\mu:=1+T(d-1, n)$. This estimate can be proved by induction using formula (3.6) (in which we drop the "t"). To make this clearer, if $X^{\beta}=X_{1} X^{\alpha}$ with $|\alpha|=d+k$, then the coefficient of $X^{\tilde{\delta}}$ in $H_{S}\left(X^{\beta}\right)$ is given by

$$
\sum_{|\delta|=d} C_{\delta}\left(X^{\alpha}\right) C_{\tilde{\delta}}\left(X_{1} X^{\delta}\right)+C_{\left(\tilde{\delta}_{1}-1, \tilde{\delta}_{2}, \ldots\right)}\left(X^{\alpha}\right) \quad \text { or only } \sum_{|\delta|=d} C_{\delta}\left(X^{\alpha}\right) C_{\tilde{\delta}}\left(X_{1} X^{\delta}\right)
$$

according as $\tilde{\delta}_{1} \geq 1$ or not. Therefore we deduce

$$
\left\|H_{S}\left(X^{\beta}\right)\right\|_{\infty} \leq\left(\sum_{|\delta|=d} M_{S} M_{k}\right)+M_{k} \leq M_{k+1}
$$


Lemma 4.3. Let $Y=\left\{y^{0}, \ldots, y^{m}\right\}$ be a subset formed of $m+1$ points in $\mathbb{R}^{n}$. We set $|Y|:=\operatorname{Max}\left\{\left|y^{i}\right|, i=0, \ldots, m\right\}$. Then for every function $f$ of class $C^{m+1}$ on a neighborhood of $C v(Y)$ we have

$$
\left\|K_{Y}(f)-\mathcal{T}^{m} f\right\|_{\infty} \leq O_{m, n}(|Y|) .\|f\|_{m+1} .
$$

where $\|f\|_{m+1}=\|f\|_{m+1, C v(Y)}$ and as above $C v(Y)$ is the convex hull of $Y \cup\{0\}$.

Proof. Since the polynomials $K_{Y} f-\mathcal{T}^{m} f$ (as well as the polynomials $Q_{\alpha}$ defined below) have real coefficients, we may consider them as polynomials of $n$ complex variables. Let $\mathbb{P}:=\left\{z=\left(z_{1}, \ldots, z_{n}\right),\left|z_{i}\right| \leq 1, i=1, \ldots, n\right\}$ be the unit polydisc in $\mathbb{C}^{n}$. Here $\left|z_{i}\right|$ is the modulus of the complex number $z_{i}$. We shall prove that

$$
\max _{z \in \mathbb{P}}\left|K_{Y}(f, z)-\mathcal{T}^{m}(f, z)\right| \leq O_{m, n, d}(|Y|)\|f\|_{m+1}
$$

from which, using the Cauchy inequalities (see e.g. [H, 2.2.7]), the estimates to be proved follow. Just to shorten somewhat the formulas we shall write for $t \in \Delta_{i}$,

$$
R_{i}(t):=y^{0}+\sum_{j=1}^{i} t_{j}\left(y^{j}-y^{0}\right) \in C v(Y)
$$

First step. There exist polynomials $Q_{\alpha}$ such that

$$
K_{Y} f=f\left(y^{0}\right)+\sum_{i=1}^{m} \sum_{|\alpha|=i}\left\{\int_{\Delta_{i}} D^{\alpha} f\left(R_{i}(t)\right) d m(t)\right\} Q_{\alpha} .
$$

Furthermore the polynomials $Q_{\alpha}$ are defined, when $|\alpha|=i$, by the relation

$$
\left\langle x-y^{0}, \lambda\right\rangle \ldots\left\langle x-y^{i-1}, \lambda\right\rangle=\sum_{|\alpha|=i} Q_{\alpha}(x) \lambda^{\alpha} .
$$

This formula is known (see [B, (3.4.2)] or [C, Lemma 4.3]). The existence of the polynomials $Q_{\alpha}$ follows immediately from the formula (3.7) and that they verify the formula (4.3) can be seen on applying (4.2) to functions of the form $f=$ $g(\langle x, \lambda\rangle)$ taking into account the fundamental property of Kergin interpolants and the classical one-variable Newton formula.

Second step. We claim that for every $z \in \mathbb{P}$ and for $|\alpha|=i \geq 1$ we have

$$
\begin{aligned}
\left|Q_{\alpha}(z)\right| & \leq(n+|Y|)^{i} \\
\left|Q_{\alpha}(z)-z^{\alpha}\right| & \leq i|Y|(n+|Y|)^{i-1} .
\end{aligned}
$$

For $w$ a complex number and $\lambda \in \mathbb{C}^{n}$, we let

$$
p_{\lambda}(w):=\left(w-\left\langle y^{0}, \lambda\right\rangle\right) \ldots\left(w-\left\langle y^{i-1}, \lambda\right\rangle\right)
$$

then we have, for $|w| \leq n, \lambda \in \mathbb{P}$

$$
\left|p_{\lambda}(w)-w^{i}\right| \leq \sum_{j=0}^{i-1}\left(\begin{array}{l}
i \\
j
\end{array}\right)(|Y|)^{i-j} w^{j}=(w+|Y|)^{i}-w^{i} \leq i|Y|(n+|Y|)^{i-1} .
$$

Now setting $w=\langle z, \lambda\rangle$ we see that $|w| \leq n$ whenever $z$ and $\lambda$ belong to $\mathbb{P}$ so that

$$
\left|\sum_{|\alpha|=i}\left(Q_{\alpha}(z)-z^{\alpha}\right) \lambda^{\alpha}\right|=\left|p_{\lambda}(\langle z, \lambda\rangle)-\langle z, \lambda\rangle^{i}\right| \leq i|Y|(n+|Y|)^{i-1}
$$

Using the Cauchy inequalities we obtain the second estimate in (4.4). The first one is proved similarly. 
Conclusion.

$$
\begin{array}{r}
K_{Y}(f, z)-\mathcal{T}^{m}(f, z) \\
=f\left(y^{0}\right)-f(0)+\sum_{i=1}^{m} \sum_{|\alpha|=i}\left\{\int _ { \Delta _ { i } } \left[D^{\alpha} f\left(R_{i}(t)\right)-\right.\right. \\
\left.D^{\alpha} f(0)\right] d m(t) Q_{\alpha}(z) \\
\left.+\frac{D^{\alpha} f(0)}{\alpha !}\left(Q_{\alpha}(z)-z^{\alpha}\right)\right\},
\end{array}
$$

whence, using (4.4), the mean value theorem, and that $m\left(\Delta_{i}\right)=\frac{1}{i !}$, we obtain for $z \in \mathbb{P}$

$$
\begin{aligned}
& \left|K_{Y}(f, z)-\mathcal{T}^{m}(f, z)\right| \\
& \leq|Y| .\|f\|_{1}+\sum_{i=1}^{m} \sum_{|\alpha|=i} \frac{\|f\|_{i+1}}{i !}|Y|(n+|Y|)^{i}+\frac{\|f\|_{i}}{\alpha !} i|Y|(n+|Y|)^{i-1} \\
& \leq|Y|\|f\|_{m+1}(1+n|Y|) e^{n(n+|Y|)}=O_{m, n}(|Y|)\|f\|_{m+1}
\end{aligned}
$$

and the lemma follows.

Proof of 4.1. We use the notation (3.1) for $S$ and the same auxiliary interpolation polynomials as in the proof of Theorem 3.3. Namely, with the same notation as there (dropping the " $t$ "), $K:=K_{Y}$ and $\tilde{K}:=K_{W}$. Then we have, see (3.10) and $(3.11)$

$$
H_{S} f-\mathcal{T}^{d} f=\left[H_{S}(K f)-\mathcal{T}^{d}(K f)\right]+\left[\mathcal{T}^{d}(K f)-\tilde{K}(K f)\right]+\left[\tilde{K} f-\mathcal{T}^{d} f\right]
$$

whence, by Lemmas 4.2 and 4.3

$$
\begin{aligned}
& \left\|H_{S} f-\mathcal{T}^{d} f\right\|_{\infty} \\
& \quad \leq\left\|H_{S}(K f)-\mathcal{T}^{d}(K f)\right\|_{\infty}+\left\|\mathcal{T}^{d}(K f)-\tilde{K}(K f)\right\|_{\infty}+\left\|\tilde{K} f-\mathcal{T}^{d} f\right\|_{\infty} \\
& \quad \leq O\left(M_{S}\right) .\|K f\|_{\infty}+O(|S|) .\left(\|K f\|_{d+1}+\|f\|_{d+1} \|\right) .
\end{aligned}
$$

Thus it remains to verify that $\|K f\|_{\infty}$ and $\|K f\|_{d+1}$ are both $O\left(\|f\|_{l}\right)$. Using (4.2), it is easily seen that $\|K f\|_{\infty}=O\left(\|f\|_{l-1}\right)$ and $\|K f\|_{d+1}=O\left(\|f\|_{l}\right)$, we shall omit the details. As for the precise computation of the constant, it suffices to note that the estimates $\left\|Q_{\alpha}\right\|_{\infty} \leq(n+|S|)^{|\alpha|}$ follow from the proof of Lemma 4.3 and that we could prove along the same lines that $\left\|Q_{\alpha}\right\|_{l}=\left\|Q_{\alpha}\right\|_{d+1} \leq|\alpha| !(n+|S|)^{|\alpha|}$.

We conclude this section by pointing to a bound for $|S|$ in terms of $M_{S}$ that was more or less apparent in Lemma 3.4. Let us assume e.g. that $a \in S \Longrightarrow|a|<1 / 2$, then for $a \in S$ and $j \in\{1, \ldots, n\}$, we have, again with the notation (3.1)

$$
\begin{gathered}
\left|a_{j}\right|^{d+1}=\left|H_{S}\left(X_{j}^{d+1}, a\right)\right| \leq M_{S} \sum_{|\alpha| \leq d} a^{\alpha} \leq M_{S} \cdot 2^{n} \\
\Longrightarrow|S| \leq n 2^{\frac{n}{d+1}} \cdot M_{S}^{\frac{1}{d+1}} .
\end{gathered}
$$




\section{Two EXAMPles}

Example 5.1. We shall work in $\mathbb{R}^{2}$ with coordinates $(x, y)$. Let $a=\left(a_{1}, a_{2}\right)$ and $b=\left(b_{1}, b_{2}\right) \in \mathbb{R}^{2}$. For every function $f$ defined, and with appropriate derivatives at $a, b$ we look for a polynomial $p$ of degree 2 satisfying the following six conditions:

$$
\begin{aligned}
& p(0)=f(0), \quad \frac{\partial p}{\partial x}(0)=\frac{\partial f}{\partial x}(0), \quad \frac{\partial p}{\partial y}(0)=\frac{\partial f}{\partial y}(0), \\
& f(a)=p(a), \quad \frac{\partial p}{\partial x}(a)=\frac{\partial f}{\partial x}(a), \quad f(b)=p(b) .
\end{aligned}
$$

In other words, we are considering the interpolation scheme of degree 2 in $\mathbb{R}^{2}$ (and $\left.p=H_{S}(f)\right)$ defined by

$$
S:=\{(0,\{(0,0)(1,0),(0,1)\}) ;(a,\{(0,0),(1,0)\}),(b,\{(0,0)\})\} .
$$

Let us first study under what condition(s), (5.2) is really a well defined interpolation scheme. Let

$$
p(x, y)=c_{1}+c_{2} x+c_{3} y+c_{4} x^{2}+c_{5} x y+c_{6} y^{2},
$$

then the coefficients $c_{i}=c_{i}(f)$ are required to satisfy

$$
\left(\begin{array}{cccccc}
1 & 0 & 0 & 0 & 0 & 0 \\
0 & 1 & 0 & 0 & 0 & 0 \\
0 & 0 & 1 & 0 & 0 & 0 \\
1 & a_{1} & a_{2} & a_{1}^{2} & a_{1} a_{2} & a_{2}^{2} \\
0 & 1 & 0 & 2 a_{1} & a_{2} & 0 \\
1 & b_{1} & b_{2} & b_{1}^{2} & b_{1} b_{2} & b_{2}^{2}
\end{array}\right)\left(\begin{array}{c}
c_{1} \\
c_{2} \\
c_{3} \\
c_{4} \\
c_{5} \\
c_{6}
\end{array}\right)=\left(\begin{array}{c}
f(0) \\
\partial f / \partial x(0) \\
\partial f / \partial y(0) \\
f(a) \\
\partial f / \partial x(a) \\
f(b)
\end{array}\right) .
$$

Therefore we obtain immediately

Property 5.2. (5.1) is an interpolation scheme of degree 2 in $\mathbb{R}^{2}$ if and only if

$$
V=V(a, b):=\left|\begin{array}{ccc}
a_{1}^{2} & a_{1} a_{2} & a_{2}^{2} \\
2 a_{1} & a_{2} & 0 \\
b_{1}^{2} & b_{1} b_{2} & b_{2}^{2}
\end{array}\right| \neq 0 .
$$

We shall now consider the family of scheme $S_{t}$ (for notational convenience, we shall consider here $t \rightarrow 0$ ) on taking

$$
\left\{\begin{array}{cl}
a_{1}=a_{1}^{t}:=t \bar{a}_{1}, & b_{1}=b_{1}^{t}:=t \bar{b}_{1}, \\
a_{2}=a_{2}^{t}:=t^{\alpha} \bar{a}_{2}, & b_{2}=b_{2}^{t}:=t^{\alpha} \bar{b}_{2},
\end{array}\right.
$$

where $\bar{a}=\left(\bar{a}_{1}, \bar{a}_{2}\right), \bar{b}=\left(\bar{b}_{1}, \bar{b}_{2}\right)$ and $\alpha>0$ will be fixed later.

We note that with these definitions, the determinant

$$
V=2 a_{1} a_{2}^{2} b_{1} b_{2}-a_{2}^{3} b_{1}^{2}-a_{1}^{2} a_{2} b_{2}^{2}
$$

is homogeneous of degree $(2+3 \alpha)$ in $t$, i.e.,

$$
V(a, b)=t^{2+3 \alpha} V(\bar{a}, \bar{b}) .
$$

We now investigate the condition (3.2) of Theorem 3.3. For this interpolation scheme, we just have to study if, for the polynomials $q(x, y)=x^{3}, y^{3}, x^{2} y, x y^{2}$, the 
three coefficients

$$
\begin{aligned}
c_{4}(q)= & \frac{1}{V}\left|\begin{array}{ccc}
q(a) & a_{1} a_{2} & a_{2}^{2} \\
\partial q / \partial x(a) & a_{2} & 0 \\
f(b) & b_{1} b_{2} & b_{2}^{2}
\end{array}\right| ; \quad c_{5}(q)=\frac{1}{V}\left|\begin{array}{ccc}
a_{1}^{2} & q(a) & a_{2}^{2} \\
2 a_{1} & \partial q / \partial x(a) & 0 \\
b_{1}^{2} & q(b) & b_{2}^{2}
\end{array}\right| ; \\
& \text { and } c_{6}(q)=\frac{1}{V}\left|\begin{array}{ccc}
a_{1}^{2} & a_{1} a_{2} & q(a) \\
2 a_{1} & a_{2} & \partial q / \partial x(a) \\
b_{1}^{2} & b_{1} b_{2} & q(b)
\end{array}\right|
\end{aligned}
$$

tend to 0 as $t$ approaches $0\left(c_{1}, c_{2}, c_{3}\right.$ being identically zero).

We can verify that the numerators of $c_{4}, c_{5}, c_{6}$, say $D_{4}, D_{5}, D_{6}$ are all homogeneous in a power of $t$. This power is indicated in the following array:

\begin{tabular}{c|c|c|c|} 
& $D_{4}$ & $D_{5}$ & $D_{6}$ \\
\hline$x^{3}$ & $t^{3+3 \alpha}$ & $t^{4+2 \alpha}$ & $t^{5+\alpha}$ \\
$x^{2} y$ & $t^{2+4 \alpha}$ & $t^{3+3 \alpha}$ & $t^{4+2 \alpha}$ \\
$x y^{2}$ & $t^{5 \alpha+1}$ & $t^{4 \alpha+2}$ & $t^{3+3 \alpha}$ \\
$y^{3}$ & $t^{6 \alpha}$ & $t^{5 \alpha+1}$ & $t^{2+4 \alpha}$
\end{tabular}

We can now prove the

Proposition 5.3. Let us consider the family of schemes $S_{t}$ in which $\alpha, \bar{a}$ and $\bar{b}$ are fixed in order that

(i) $\alpha \in(3 / 2,2)$;

(ii) $V(\bar{a}, \bar{b}) \neq 0$ (see $(5.4)), \bar{a}_{1} \neq 0, \bar{a}_{1} \neq \bar{b}_{1}$;

(iii) The coefficient $D_{6}$ for $x^{3}$ is zero for $t=1$ (and therefore for every $t$ ), specifically, this means that one has

$$
\bar{a}_{1}^{2} \bar{b}_{1}\left\{-\bar{a}_{1}^{2} \bar{b}_{2}+2 \bar{a}_{1} \bar{a}_{2} \bar{b}_{1}-\bar{a}_{2} \bar{b}_{1}^{2}\right\}=0 .
$$

Then conditions (3.2) of Theorem 3.3 are verified. Consequently, since o $\left(S_{t}\right)=5$, for every function $f$ of class $C^{4}$ in a neighborhood of the origin we have

$$
\lim _{t \rightarrow 0} H_{S_{t}} f=\mathcal{T}^{2} f .
$$

Furthermore, the conditions are optimal in the sense that one can find functions of class $C^{3}$ for which the convergence does not hold.

Proof. First we note there exist points $\bar{a}$ and $\bar{b}$ satisfying (ii) and (iii). One can take e.g. $\bar{a}_{1}=1, \bar{a}_{2}=2, \bar{b}_{1}=2, \bar{b}_{2}=0$. Now, by (5.4) and (5.6), the $3 \times 4=12$ coefficients $c_{i}=D_{i} / V$ are homogeneous in various powers of $t$. When $\alpha \in(3 / 2,2)$ we easily check that all of these powers are $>0$ with only one exception, the one in the $c_{6}$ coefficient for $x^{3}$. However, by (iii), this coefficient is constantly equal to zero. Therefore we can conclude that $M_{S_{t}}$ tends to 0 as $t \rightarrow 0$ so that Theorem 3.3 applies.

As for the optimality of the required smoothness, let us consider the function $f$ defined by

$$
f(x, y)=x^{\lambda}
$$

where $\lambda \in(3,2 \alpha)$ and $\lambda$ is a rational number of the form $\frac{p}{q}$ with $p, q$ odd. Then $f \in C^{3}\left(\mathbb{R}^{2}\right)$. Now, the coefficient $c_{6}$ for $f$ is of the form

$$
c_{6}=c_{6}(f):=u(\lambda) \frac{1}{V(\bar{a}, \bar{b})} \frac{t^{2+\lambda+\alpha}}{t^{2+3 \alpha}}
$$


with

$$
u(\lambda)=\left|\begin{array}{cc}
2 \bar{a}_{1} & \bar{a}_{2} \\
\bar{b}_{1}^{2} & \bar{b}_{1} \bar{b}_{2}
\end{array}\right| \bar{a}_{1}^{\lambda}-\left|\begin{array}{cc}
\bar{a}_{1}^{2} & \bar{a}_{1} \bar{a}_{2} \\
\bar{b}_{1}^{2} & \bar{b}_{1} \bar{b}_{2}
\end{array}\right| \lambda\left|\bar{a}_{1}\right|^{\lambda-1}+\left|\begin{array}{cc}
\bar{a}_{1}^{2} & \bar{a}_{1} \bar{a}_{2} \\
2 \bar{a}_{1} & \bar{a}_{2}
\end{array}\right| \bar{b}_{1}^{\lambda}
$$

Since $V(\bar{a}, \bar{b}) \neq 0$ the three $2 \times 2$ determinants above cannot all vanish. Hence, since $\bar{a}_{1} \neq 0$ and $\bar{a}_{1} \neq \bar{b}_{1}, u(\lambda)$ has only finitely many roots. Therefore one can find a rational $\lambda$ of the form indicated such that $c_{6} \neq 0$ and is therefore unbounded as $t \rightarrow \infty$. Consequently, $H_{S_{t}} f$ does not converge to $\mathcal{T}^{2} f(=0)$ as $t \rightarrow 0$.

Example 5.4. Using the same idea as the previous example, we construct a family $S_{t}(t \rightarrow 0)$ of unisolvent arrays of degree 2 in $\mathbb{R}^{2}$ of the form $A_{t}(S)$ which violates the hypothesis of Corollary 2.2 but satisfies condition (3.2) of Theorem 3.3. Hence the convergence to the Taylor polynomial is verified for functions of class $C^{5}$ in a neighborhood of the origin. In a particular case, we shall exhibit a function of class $C^{3}$ (here, $d=2$ and $3=d+1$ ) for which $L_{S_{t}} \nrightarrow \mathcal{T}^{d} f$.

Let us take a unisolvent array (to be fixed later)

$$
S:=\left\{\bar{a}^{1}, \bar{a}^{2}, \ldots, \bar{a}^{6}\right\}
$$

and

$$
S_{t}=\left\{a_{t}^{1}, a_{t}^{2}, \ldots, a_{t}^{6}\right\}:=A_{t}(S), \quad \text { with } \quad A_{t}=\left(\begin{array}{cc}
t & 0 \\
0 & t^{\alpha}
\end{array}\right)
$$

where $\alpha$ is again to be fixed later.

We write

$$
L_{S_{t}} f:=c_{1}+c_{2} x+c_{3} y+c_{4} x^{2}+c_{5} x y+c_{6} y^{2},
$$

as, in 5.5, the coefficients $c_{i}=c_{i}(f)$ are given by the usual Cramer formulas. For instance, we have, dropping the " $\mathrm{t}$ ",

$$
c_{5}=\frac{1}{V D M\left(a^{1}, \ldots, a^{6}\right)}\left|\begin{array}{cccccc}
1 & a_{1}^{1} & a_{2}^{1} & \left(a_{1}^{1}\right)^{2} & f\left(a^{1}\right) & \left(a_{2}^{1}\right)^{2} \\
1 & a_{1}^{2} & a_{2}^{2} & \left(a_{1}^{2}\right)^{2} & f\left(a^{2}\right) & \left(a_{2}^{2}\right)^{2} \\
\ldots & \ldots & \ldots & \ldots \ldots \ldots \ldots \ldots \ldots \ldots \ldots \ldots \\
\ldots & \ldots & \ldots & \ldots \ldots \ldots \ldots \ldots \ldots \ldots \ldots \\
1 & a_{1}^{6} & a_{2}^{6} & \left(a_{1}^{6}\right)^{2} & f\left(a^{6}\right) & \left(a_{2}^{6}\right)^{2}
\end{array}\right| .
$$

Now the Vandermonde $\operatorname{VDM}\left(a^{1}, \ldots, a^{6}\right)$ is homogeneous in a power of $t$, namely

$$
\operatorname{VDM}\left(a^{1}, \ldots, a^{6}\right)=t^{4+4 \alpha} V D M\left(\bar{a}^{1}, \ldots, \bar{a}^{6}\right)
$$

and similarly for the polynomials $q(x, y)=x^{3}, y^{3}, x^{2} y, x y^{2}$ the different numerators (say $D_{i}$ ) of the coefficients $c_{i}$ are homogeneous in a power of $t$. This power is indicated in the next array.

\begin{tabular}{c|c|c|c|c|c|c} 
& $D_{1}$ & $D_{2}$ & $D_{3}$ & $D_{4}$ & $D_{5}$ & $D_{6}$ \\
\hline$x^{3}$ & $t^{7+4 \alpha}$ & $t^{6+4 \alpha}$ & $t^{7+3 \alpha}$ & $t^{5+4 \alpha}$ & $t^{6+3 \alpha}$ & $t^{7+2 \alpha}$ \\
$x^{2} y$ & $t^{6+5 \alpha}$ & $t^{5+5 \alpha}$ & $t^{6+4 \alpha}$ & $t^{4+5 \alpha}$ & $t^{5+4 \alpha}$ & $t^{6+3 \alpha}$ \\
$x y^{2}$ & $t^{5+6 \alpha}$ & $t^{4+6 \alpha}$ & $t^{5+5 \alpha}$ & $t^{3+6 \alpha}$ & $t^{4+5 \alpha}$ & $t^{5+4 \alpha}$ \\
$y^{3}$ & $t^{4+7 \alpha}$ & $t^{3+7 \alpha}$ & $t^{4+6 \alpha}$ & $t^{2+7 \alpha}$ & $t^{3+6 \alpha}$ & $t^{4+5 \alpha}$
\end{tabular}

Proposition 5.5. Let us consider the family $S_{t}(t \rightarrow 0)$ of unisolvent arrays of degree 2 in which $\alpha, \bar{a}^{1}, \ldots, \bar{a}^{6}$ are fixed in order that

(i) $\alpha \in\left(\frac{3}{2}, 2\right)$;

(ii) $\operatorname{VDM}\left(\bar{a}^{1}, \ldots, \bar{a}^{6}\right) \neq 0$; 
(iii) The coefficient $c_{6}$ for $q(x, y)=x^{3}$ is zero for $t=1$ (and therefore for every t).

Then the family $S_{t}$ satisfies the condition (3.2) of Theorem 3.3 but not the condition of 2.2.

Proof. Let us first prove that there exist points satisfying the required conditions. Let us take for example

$$
\bar{a}_{2}^{1}=\bar{a}_{2}^{2}=\bar{a}_{2}^{3}=0 ; \quad \bar{a}_{2}^{4}=\bar{a}_{2}^{5} ; \quad \bar{a}_{1}^{1}=\bar{a}_{1}^{4}=0 ;
$$

then a simple calculation shows that

$$
\begin{aligned}
V D M\left(\bar{a}^{1}, \ldots, \bar{a}^{6}\right) & =\left|\begin{array}{cccccc}
1 & 0 & 0 & 0 & 0 & 0 \\
1 & \bar{a}_{1}^{2} & 0 & \left(\bar{a}_{1}^{2}\right)^{2} & 0 & 0 \\
1 & \bar{a}_{1}^{3} & 0 & \left(\bar{a}_{1}^{3}\right)^{2} & 0 & 0 \\
1 & 0 & \bar{a}_{2}^{4} & 0 & 0 & \left(\bar{a}_{2}^{4}\right)^{2} \\
1 & \bar{a}_{1}^{5} & \bar{a}_{2}^{4} & \left(\bar{a}_{1}^{5}\right)^{2} & \bar{a}_{2}^{4} \bar{a}_{1}^{5} & \left(\bar{a}_{2}^{4}\right)^{2} \\
1 & \bar{a}_{1}^{6} & \bar{a}_{2}^{6} & \left(\bar{a}_{1}^{6}\right)^{2} & \bar{a}_{1}^{6} \bar{a}_{2}^{6} & \left(\bar{a}_{2}^{6}\right)^{2}
\end{array}\right| \\
& =\bar{a}_{2}^{6}\left(\bar{a}_{2}^{6}-\bar{a}_{2}^{4}\right)\left(\bar{a}_{2}^{4}\right)^{2} \bar{a}_{1}^{2} \bar{a}_{1}^{3} \bar{a}_{1}^{5}\left(\bar{a}_{1}^{2}-\bar{a}_{1}^{3}\right)
\end{aligned}
$$

which does not vanish if

$$
\bar{a}_{2}^{4} \neq \bar{a}_{2}^{6} ; \quad \bar{a}_{1}^{2} \neq \bar{a}_{1}^{3} ; \quad \bar{a}_{2}^{4}, \bar{a}_{2}^{6}, \bar{a}_{1}^{2}, \bar{a}_{1}^{3}, \bar{a}_{1}^{5} \neq 0 .
$$

So we fix $\bar{a}_{2}^{4}, \bar{a}_{2}^{6}, \bar{a}_{1}^{2}, \bar{a}_{1}^{3}, \bar{a}_{1}^{5}$ in order that (5.11) holds. Next, the coefficient $D_{6}$ for $x^{3}$ is, as a function of $\bar{a}_{1}^{6}$, a cubic polynomial whose leading coefficient is $\left(\bar{a}_{2}^{4}\right)^{2} \bar{a}_{1}^{5} \bar{a}_{1}^{2} \bar{a}_{1}^{3}\left(\bar{a}_{1}^{2}-\bar{a}_{1}^{3}\right)$ which by (5.11) is not zero. We can take for $\bar{a}_{1}^{6}$ any real root of this polynomial. (It is easily verified that this polynomial has always the root 0 .) We have thus constructed an array satisfying (ii) and (iii).

Now, under hypothesis (i), all the powers (in $t$ ) of coefficients $c_{i}(q)$ are positive for $q(x, y)=x^{3}, x^{2} y, x y^{2}, y^{3}$ except the coefficient $c_{6}$ for $x^{3}$ which vanishes due to hypothesis (iii). The proposition is proved.

Finally, note that, under assumption (i), we have, using the same norm as in 2.3, $\left\|A_{t}\right\|^{3}\left\|A_{t}^{-1}\right\|^{2}=t^{3} / t^{2 \alpha} \rightarrow \infty$ as $t \rightarrow 0$ so that Corollary 2.2 does not apply.

Suppose that $\left(\bar{a}^{1}, \ldots, \bar{a}^{6}\right)$ is an array of the form given in the previous proof (thus it satisfies the condition (ii) and (iii) in 5.5) and that furthermore $\bar{a}_{1}^{6}$ is not equal to $\bar{a}_{1}^{5}, \bar{a}_{1}^{3}, \bar{a}_{1}^{2}$ or zero. Then we can find a function $f$ of Class $C^{3}$ whose family of interpolants does not converge to $\mathcal{T}^{2} f$. Again we shall consider the functions

$$
f(x, y)=x^{\lambda}
$$

where $\lambda \in(3,2 \alpha)$ and $\lambda$ is a rational number of the form $\frac{p}{q}$ with $p, q$ odd.

The $c_{6}$ coefficient is of the form

$$
c_{6}=c_{6}(f):=\left|\begin{array}{cccccc}
1 & 0 & 0 & 0 & 0 & 0 \\
1 & \bar{a}_{1}^{2} & 0 & \left(\bar{a}_{1}^{2}\right)^{2} & 0 & \left(\bar{a}_{1}^{2}\right)^{\lambda} \\
1 & \bar{a}_{1}^{3} & 0 & \left(\bar{a}_{1}^{3}\right)^{2} & 0 & \left(\bar{a}_{1}^{3}\right)^{\lambda} \\
1 & 0 & \bar{a}_{2}^{4} & 0 & 0 & 0 \\
1 & \bar{a}_{1}^{5} & \bar{a}_{2}^{4} & \left(\bar{a}_{1}^{5}\right)^{2} & \bar{a}_{2}^{4} \bar{a}_{1}^{5} & \left(\bar{a}_{1}^{5}\right)^{\lambda} \\
1 & \bar{a}_{1}^{6} & \bar{a}_{2}^{6} & \left(\bar{a}_{1}^{6}\right)^{2} & \bar{a}_{1}^{6} \bar{a}_{2}^{6} & \left(\bar{a}_{1}^{6}\right)^{\lambda}
\end{array}\right| \cdot \frac{1}{V D M} \cdot \frac{t^{4+\lambda+2 \alpha}}{t^{4+4 \alpha}} .
$$

Now we claim that we can find $\lambda$ of the previous form such that $c_{6}(f)$ does not vanish. Indeed, otherwise $c_{6}(\lambda)$ would vanish identically as an analytic function of $\lambda$ and in particular the coefficient of $\left(\bar{a}_{1}^{6}\right)^{\lambda}$ in $D_{6}$ for $x^{\lambda}$ would be zero which is impossible since the coefficient is the same as that for $\left(\bar{a}_{1}^{6}\right)^{3}$ in $D_{6}$ for $x^{3}$ (except 
for the power of $t$ ). Hence $c_{6}$ is unbounded as $t \rightarrow 0$ which makes the convergence to the Taylor polynomial impossible.

As to find a concrete example of points satisfying the required condition, we may take for example $\bar{a}_{1}^{2}=1, \bar{a}_{1}^{3}=3, \bar{a}_{2}^{4}=-1, \bar{a}_{1}^{5}=2, \bar{a}_{2}^{5}=-1, \bar{a}_{2}^{6}=1$ so that

$$
D_{6}\left(x^{3}\right):=12 \bar{a}_{1}^{6}\left(\left(\bar{a}_{1}^{6}\right)^{2}-4 \bar{a}_{1}^{6}+2\right)
$$

and we choose for $\bar{a}_{1}^{6}$ a non-zero root of the quadratic polynomial above.

\section{REFERENCES}

[B] T. Bloom, Interpolation at discrete subsets of $\mathbb{C}^{n}$, Indiana J. of Math. 39 (1990), 1223-1243. MR 91k:32015

[Bo] L. Bos, On certain configurations of points in $\mathbb{R}^{n}$ which are unisolvent for polynomial interpolation, J. of Approx. Theory 64 (1991), 271-280. MR 91m:41005

[C] J. P. Calvi, Polynomial interpolation with prescribed analytic functionals, J. Approx. Theory 75 (1993), 136-156. MR 94j:41002

[CR] P.G. Ciarlet and P.A. Raviart, General Lagrange and Hermite interpolation in $\mathbb{R}^{n}$ with applications to finite element methods, Arch. Rat. Mech. Anal. 46 (1972), 177-199. MR 49:1730

[Co] C. Coatmelec, Approximation et interpolation des fonctions differentiables de plusieurs variables, Ann. Scient. Ec. Norm. Sup. 83 (1966), 271-341. MR 38:469

[H] L. Hörmander, An Introduction to Complex Analysis in Several Variables, North Holland, Amsterdam, 1990. MR 91a:32001

[K] P. Kergin, A natural interpolation of $C^{k}$ functions, J. of Approx. 29 no 4 (1980), 278-293. MR 82b: 41007

[LP] S. L. Lee and G. M. Phillips, Interpolation on the triangle and simplex, Approximation Theory, Wavelets and Applications (S. P. Singh, ed.), Kluwer Academic Publishers, 1995, pp. 177-196. MR 96f:41042

[L] R. A. Lorentz, Multivariate Birkhoff Interpolation, Lecture Notes in Mathematics no. 1516, Springer-Verlag. MR 94h:41001

[M] C. A. Micchelli, A constructive approach to Kergin interpolant in $\mathbb{R}^{k}$, Rocky Mountain J. 10 (3) (1980), 485-497. MR 84i:41002

[N] G. Nürnberger, Approximation by spline functions, Springer, Berlin, 1989. MR 90j:41025

[SX] T. Sauer and Y. Xu, A case study in multivariate Lagrange interpolation, Approximation Theory, Wavelets and Applications (S. P. Singh, ed.), Kluwer Academic Publishers, 1995, pp. 443-452. MR 96d:41036

[W] S. Waldron, Integral error formula for the scale of mean value interpolations which includes Kergin and Hakopian interpolation, Numer. Math. (to appear).

Department of Mathematics, University of Toronto, M5S 1A1, Toronto, Ontario, CANADA

E-mail address: bloom@math.toronto.edu

Laboratoire de mathématiques, UFR Mig, Université Paul Sabatier, 31062 Toulouse Cedex, France 